\title{
Periodic Stacking of 2D Charged Sheets : Self-Assembled Superlattice of Ni-Al Layered Double Hydroxide (LDH) and Reduced Graphene Oxide
}

\author{
Xiang Ge, ${ }^{1,2}$ Changdong Gu, ${ }^{l}$ Zongyou Yin, ${ }^{2}$ Xiuli Wang, ${ }^{l}$ Jiangpin $\mathrm{Tu},{ }^{l}$ and $\mathrm{Ju} \mathrm{Li}^{2} *$ \\ ${ }^{1}$ State Key Laboratory of Silicon Materials, Key Laboratory of Advanced Materials and Applications \\ for Batteries of Zhejiang Province and School of Materials Science and Engineering, Zhejiang \\ University, Hangzhou 310027, China. \\ ${ }^{2}$ Department of Nuclear Science and Engineering and Department of Materials Science and \\ Engineering, Massachusetts Institute of Technology, Cambridge, Massachusetts 02139, USA.
}

\begin{abstract}
Vertically stacked artificial 2D materials, such as van der Waals heterostructures, hold great scientific and technological promise. Stacking 2D atomic layers with stronger electrostatic forces in a controlled fashion could be more challenging. Positively charged atomic sheets of layered double hydroxide (LDH) such as hydrotalcite mineral with weakly bound anions have known intercalation and anion exchange properties, while reduced graphene oxide (rGO) are known to be negatively charged. So self-assembly of periodic $(\mathrm{LDH} / \mathrm{rGO})_{n}$ superlattice is possible, although true periodicity at atomic scale has never been demonstrated for Ni-Al LDH. This work introduces a new protocol for the synthesis of true $\mathrm{Ni}-\mathrm{Al} \mathrm{LDH} / \mathrm{GO}$ superlattice and the corresponding reduced product $\mathrm{Ni}-\mathrm{Al}$ $\mathrm{LDH} / \mathrm{rGO}$, by systematically optimizing various key experimental parameters in chemical exfoliation, dispersion and self-assembly by co-feeding. This method is further applied to the successful synthesis of more complex Ni-Co-Al/GO superlattice. The Ni-Al LDH/rGO superlattice is then tested as cathode in alkaline hybrid supercapacitor, with $129 \mathrm{Ah} / \mathrm{kg}$ capacity at 8-min discharge, two times that of pristine $\mathrm{Ni}-\mathrm{Al} \mathrm{LDH}$, and maintains $72.7 \%$ of its initial capacity after 10,000 charge/discharge cycles. Our superlattice synthesis strategy and its energy applications demonstrate the potential to design artificial 2D materials.
\end{abstract}

Keywords: Layered double hydroxide; grapheme; exfoliation; superlattice; hybrid supercapacitor. 


\section{Introduction}

Layered double hydroxides (LDHs) are lamellar crystals with positively charged brucite-like host layers and weakly bound anions to compensate the charge. ${ }^{1,2}$ LDHs have tunable chemical composition, redox state, intercalated anions, etc., and hence the potential to satisfy the needs in different applications. ${ }^{3-6}$ Based on Faradaic electrochemical reaction, LDHs containing electro-active species should theoretically have large specific capacity and good powder density in aqueous alkaline electrolytes. ${ }^{7}$ However, the achieved capacity of LDHs is often far below $(1 / 10 \sim 1 / 5)$ its theoretical capacity due to poor electronic conductivity. ${ }^{8-12}$ Coupling a single layer of LDH with a single layer of conductive atomic sheet can be an ideal way to facilitate the transfer of electrons and ions. Unlike van der Waals heterostructures, LDH layers are positively charged, and thus have stronger interactions of electrostatic nature. ${ }^{13}$ If two positively and negatively charged layered materials are exfoliated as monolayers respectively and dispersed as colloidal solution, it is theoretically possible to assemble them into a periodic superlattice structure. ${ }^{13,14}$ However, only a few successful cases have been reported for synthesizing superlattice of LDHs coupled with another conductive layered material like reduced graphene oxide (rGO) or conductive polymer ${ }^{3,6,15}$ based on this exfoliation-restacking strategy. Interestingly, there are also contradictory results showing that LDH and graphene based superlattice is not obtainable by exfoliation-restacking. ${ }^{16-18}$ In particular, there is no report on the successful synthesis of true Ni-Al LDH/GO superlattice (meaning superlattice diffraction in X-ray) to date, as far as we know. ${ }^{17}$ This implies that fabricating a superlattice with Ni-Al LDH and GO is much more challenging than Co-Al or Co-Ni based LDH. The reason might be related to the fact that $\mathrm{Ni}-\mathrm{Al} \mathrm{LDH}$ is intrinsically less likely to form large-sized 2D sheets compared to $\mathrm{Co}-\mathrm{Al} \mathrm{LDH},{ }^{19}$ which would reduce the probability of face-to-face interaction in the self-assembly. Therefore, to exploit the unique properties of 2D superlattice structure for $\mathrm{Ni}-\mathrm{Al} \mathrm{LDH}$, it is necessary to gain insight into how to control the assembly process. ${ }^{20}$

This work introduces a co-feeding protocol to controllably self-assemble true Ni-Al LDH/GO superlattice. We provide unambiguous X-ray diffraction evidence for the formation of ..ABAB.. stacked Ni-Al LDH (A) / graphene oxide (B) superlattice. The influences of various experimental parameters (method of feeding the precursor, solvent system, concentration, etc.) are investigated and discussed, which shed light on how to enhance the efficiency of superlattice self-assembly of 2D charged sheets. The protocol is demonstrated to be applicable to synthesizing more complex Ni-Co-Al/GO superlattice. Furthermore, unlike previous works which only used the obtained superlattice materials in wet state, ${ }^{3,6,15}$ our Ni-Al LDH/GO can be recovered in powder form by freeze-drying without aggregation or destruction of structure. The material can also endure water washing, freeze-drying and hydrazine vapor reduction. This makes our dry powder material 
compatible with most industrial plant processing. As a proof of concept, we have fabricated a high-rate electrochemical energy storage device using $\mathrm{Ni}-\mathrm{Al} / \mathrm{rGO}$ superlattice vs. activated carbon (AC) with high mass loading ( $\sim 5 \mathrm{mg} \mathrm{Ni}-\mathrm{Al} \mathrm{LDH} / \mathrm{rGO}$ and $\sim 15 \mathrm{mg} \mathrm{AC})$ in a $\sim 1 \mathrm{~cm}^{2}$ area coin cell. The full-cell device could maintain $67.4 \%$ of its initial capacitance after 10000 charge/discharge cycles.

\section{Experimental Method}

\subsection{Preparation of crystalline Ni-Al LDH with subsequent ion exchange treatment.}

The highly crystalline Ni-Al $\mathrm{LDH}$ with $\mathrm{CO}_{3}{ }^{2-}$ counter ions was first prepared using a hydrothermal process. ${ }^{19}$ Briefly, an aqueous solution containing $\mathrm{NiCl}_{2}(0.1 \mathrm{M}), \mathrm{AlCl}_{3}(0.05 \mathrm{M})$ and urea $(0.15 \mathrm{M})$ was transferred into a Teflon-lined autoclaved and sealed in a steel container. The solution was then kept at $190{ }^{\circ} \mathrm{C}$ for two days. The precipitate was purified with repeated washing and centrifugation before drying at $60{ }^{\circ} \mathrm{C}$ in an oven. For the synthesis of Ni-Co-Al- $\mathrm{CO}_{3}{ }^{2-}$, the aqueous solution contained $\mathrm{CoCl}_{2}(0.05 \mathrm{M}), \mathrm{NiCl}_{2}(0.05 \mathrm{M}), \mathrm{AlCl}_{3}(0.05 \mathrm{M})$ and urea $(0.15 \mathrm{M})$. The hydrothermal temperature was $150{ }^{\circ} \mathrm{C}$, with all other parameters the same.

A two-step ion-exchange process was then used to treat the as-prepared $\mathrm{Ni}-\mathrm{Al} \mathrm{LDH}-\mathrm{CO}_{3}{ }^{2-} \cdot 1.5 \mathrm{~g}$ $\mathrm{Ni}-\mathrm{Al} \mathrm{LDH}-\mathrm{CO}_{3}{ }^{2-}$ was added into $1.2 \mathrm{~L}$ aqueous solution containing $5 \mathrm{mM} \mathrm{HCl}$ and $1.5 \mathrm{M} \mathrm{NaCl}$. The system was magnetically stirred at $650 \mathrm{rpm}$ for 1 day. The Ni-Al LDH-Cl${ }^{-}$was separated and purified with repeated centrifugation and washing. The product was then freeze dried. To further prepare $\mathrm{Ni}-\mathrm{Al} \mathrm{LDH}$ inserted with $\mathrm{NO}_{3}^{-}, 1 \mathrm{~g} \mathrm{Ni}-\mathrm{Al} \mathrm{LDH}-\mathrm{Cl}^{-}$was added into $1 \mathrm{~L}$ aqueous solution containing $0.15 \mathrm{M} \mathrm{NaNO}_{3}$ and then magnetically stirred at $650 \mathrm{rpm}$ for 1 day. The $\mathrm{Ni}-\mathrm{Al} \mathrm{LDH}-\mathrm{NO}_{3}{ }^{-}$was also separated and purified with repeated centrifugation and washing, and then freeze dried.

\subsection{Preparation of exfoliated Ni-Al LDH in formamide and exfoliated GO in DMF}

The exfoliation of Ni-Al LDH was achieved in formamide using a mechanical shaker. Typically, $30 \mathrm{mg} \mathrm{Ni}-\mathrm{Al} \mathrm{LDH}-\mathrm{NO}_{3}{ }^{-}$was mixed with $100 \mathrm{~mL}$ formamide in a conical beaker. The beaker was vigorously shaken on a mechanical shaker at a speed of $120 \mathrm{rpm}$ for $60 \mathrm{~h}$. The resulting colloidal solution was further centrifuged at $4000 \mathrm{rpm}$ for $15 \mathrm{~min}$. The supernatant was collected.

For the preparation of exfoliated GO, we first prepared GO powder using a modified Hummer method..$^{21}$ DMF was then selected as the solvent to exfoliate GO instead of commonly used water, which is a key point of our new process. Typically, $15 \mathrm{mg}$ GO powder was exfoliated in $50 \mathrm{~mL}$ DMF with intensive sonication treatment ( $~ 8 \mathrm{~h})$. Thereafter, high-speed centrifugation (12000 rpm, $10 \mathrm{~min})$ is used to separate unexfoliated GO. The supernatant solution was collected and then diluted to 100 $\mathrm{mL}$ using formamide under stirring. 


\subsection{The co-feeding protocol for self-assembly of the Ni-Al LDH/GO superlattice}

We find that the preparation of $\mathrm{Ni}-\mathrm{Al} \mathrm{LDH} / \mathrm{GO}$ superlattice needs delicate control of the experimental condition. In a typical process, a beaker containing $200 \mathrm{~mL}$ formamide was stirred with a magnetic stirrer. $100 \mathrm{~mL} \mathrm{Ni-Al} \mathrm{LDH} \mathrm{and} 100 \mathrm{~mL}$ GO solutions were controllably dropped into the solution simultaneously, at the same speed of $1.5 \mathrm{~mL} / \mathrm{min}$ using a peristaltic pump. After the two feed solutions were completely added, the mixed solution was then stirred for another $2 \mathrm{~h}$. The flocculated product was purified with repeated centrifugation and washing. The material was first washed once with ethanol and then twice with water. The product was finally recovered with freeze-drying. For reduction, $35 \mathrm{mg} \mathrm{Ni}-\mathrm{Al} \mathrm{LDH} / \mathrm{GO}$ was placed in a container. $500 \mu \mathrm{L}$ hydrazine $(85 \%)$ in a separate vessel was put in the container, with no direct contact with Ni-Al LDH/GO. The container was sealed and kept at $90{ }^{\circ} \mathrm{C}$ for $12 \mathrm{~h}$.

\subsection{Characterizations of Synthesized Materials}

Structural characterizations: Phase and morphology were examined with X-ray diffraction (XRD, RigakuD/Max-3B), scanning electron microscope (SEM, Hitachi S-4800), and transmission electron microscope (TEM, JEOL JEM-2100 at $200 \mathrm{kV}$ ). HAADF-mapping image was obtained using TEM (FEI, Tecnai G2 F20 at $200 \mathrm{kV}$ ). The thickness of exfoliated nanosheets was characterized on Atomic force microscope (AFM, VEECO, Multimode). FTIR characterization was performed on Bruker spectrometer (TENSOR 27). Raman spectra were obtained on DXR SmartRaman (ThermoFisher).

Electrochemical characterizations: The working electrodes were prepared by a slurry-coating procedure. The slurry consisted of $85 \mathrm{wt} \%$ active materials, $5 \mathrm{wt} \%$ acetylene black (AB) and $10 \mathrm{wt} \%$ poly(vinylidene fluoride) (PVDF) dissolved in N-methyl pyrrolidinone (NMP). Then it is incorporated on nickel foams. To characterize the active material in a three-electrode system, the working electrode is a $1 \mathrm{~cm} \times 3 \mathrm{~cm}$ nickel foam loaded with $\sim 1 \mathrm{mg}$ active material. A Pt foil is used as the counter electrode. $\mathrm{A} \mathrm{Hg} / \mathrm{HgO}$ electrode immersed in $1 \mathrm{M} \mathrm{KOH}$ was connected with a salt bridge as the reference electrode. The electrolyte is a $6 \mathrm{M} \mathrm{KOH}$ aqueous solution.

To fabricate the hybrid supercapacitor device with high loading, activated carbon (AC) was used as the negative electrode (using same slurry-coating). The loading mass is about $5 \mathrm{mg} \mathrm{Ni-Al}$ $\mathrm{LDH} / \mathrm{rGO}$ vs. $15 \mathrm{mg}$ AC. The substrates are both round nickel foam with diameter of $12 \mathrm{~mm}$. They are assembled into a coin-type cell (CR2025) using cellulose acetate (CA) as the separator.

\section{Results and discussions}

\subsection{Preparation of exfoliated nanosheets}


Fig. 1 illustrates the synthetic route of Ni-Al LDH/GO and its reduced product Ni-Al LDH/rGO at optimized condition (See details in the Experimental Method section). The synthesis of Ni-Al $\mathrm{LDH} / \mathrm{rGO}$ superlattice includes the preparation of two colloidal solutions containing exfoliated $\mathrm{Ni}-\mathrm{Al}$ LDH and GO respectively, the self-assembly using a co-feeding method, as well as the subsequent hydrazine vapor reduction to convert $\mathrm{Ni}-\mathrm{Al} \mathrm{LDH} / \mathrm{GO}$ superlattice to $\mathrm{Ni}-\mathrm{Al} \mathrm{LDH} / \mathrm{rGO}$ superlattice. LDHs with specific interlayer anions $\left(\mathrm{NO}_{3}{ }^{-}\right)$can be exfoliated in formamide while as-prepared LDHs are often intercalated with $\mathrm{CO}_{3}{ }^{2-}$. To prepare $\mathrm{Ni}-\mathrm{Al} \mathrm{LDH} /$ formamide colloid, well crystallized Ni-Al $\mathrm{LDH}-\mathrm{CO}_{3}{ }^{2-}$ was first prepared by hydrothermal method. The Ni-Al $\mathrm{LDH}-\mathrm{CO}_{3}{ }^{2-}$ was then converted to $\mathrm{Ni}-\mathrm{Al} \mathrm{LDH}_{-} \mathrm{Cl}^{-}$(as an intermediate) by an acid-salt treatment and subsequently converted to Ni-Al LDH-NO ${ }_{3}^{-}$by anion exchange. ${ }^{19}$ The XRD patterns and FTIR spectrum of the Ni-Al LDH inserted with $\mathrm{CO}_{3}{ }^{2-}, \mathrm{Cl}^{-}$or $\mathrm{NO}_{3}^{-}$(Figure S1a and S1b) shows that the exchange of inserted anions completed thoroughly. Both Ni-Al LDH- $\mathrm{CO}_{3}{ }^{2-}$ and $\mathrm{Ni}-\mathrm{Al} \mathrm{LDH}-\mathrm{NO}_{3}{ }^{-}$show similar morphologies of hexagonal plates (Figure S1c and S1d), indicating the atomic structure of the LDH was well maintained during the two-step anion-exchange process. The exfoliated Ni-Al LDH was prepared by mechanical shaking of $\mathrm{Ni}-\mathrm{Al} \mathrm{LDH}_{-} \mathrm{NO}_{3}^{-}$in formamide and purified with centrifugation. The Ni-Al LDH/formamide colloid shows clear Tyndall effect (Fig. 2a). AFM images shows the thickness of $\mathrm{Ni}-\mathrm{Al} \mathrm{LDH}$ is about $0.8(=2.5-1.7) \mathrm{nm}$, similar to the sum of basal layer of LDHs $(0.48 \mathrm{~nm})$ and that of an adsorbed monolayer $(0.3 \mathrm{~nm})$ observed for other LDHs. ${ }^{19,22}$ It is worth noting that the lateral grain size of Ni-Al LDH is about 50 200 nm (Fig. 2a), which is much smaller than those of other LDHs (Co-Al, Zn-Co-Al, Ni-Fe etc.) which range from 500 2000 nm. ${ }^{3,6,19}$ This observation may be due to the intrinsic nature that $\mathrm{Ni}-\mathrm{Al} \mathrm{LDH}$ is less anisotropic. In literature, the crystallinity of Ni-Al LDH is often worse than that of Co-Al LDH when prepared under similar conditions. ${ }^{19,22,23}$ In the self-assembly, the formation of superlattice structure requires face-to-face interactions and thus larger grain size would be preferred. If a strategy can overcome this challenge and is demonstrated to be successful to synthesize $\mathrm{Ni}-\mathrm{Al} \mathrm{LDH} / \mathrm{GO}$ superlattice, it should be rational to expect this protocol is more versatile, as we will further discuss later.

The preparation of GO follows a modified Hummer method described elsewhere. ${ }^{21}$ It is worth noting that the solvent used to exfoliate GO has a great impact on the subsequent assembly process. In the synthesis of Ni-Al LDH/GO superlattice, DMF must be used to disperse GO instead of water, which is different from the synthesis of other LDH/GO or LDH/polymer superlattice. ${ }^{3,6,15}$ After intensive ultra-sonication, high-speed centrifugation is needed to obtain well dispersed monolayer GO in DMF. The solution is further diluted with formamide. AFM image (Fig. 2b) shows that the lateral size of GO is $200 \sim 2000 \mathrm{~nm}$. The AFM thickness is about $0.7 \mathrm{~nm}$, close to the previous results. $^{21,24}$ 


\subsection{Assembly of Ni-Al LDH/GO superlattice}

The controllable self-assembly can be performed when the two types of exfoliated monolayers are well dispersed in solution. We need to emphasize that a "co-feeding" protocol is necessary to achieve

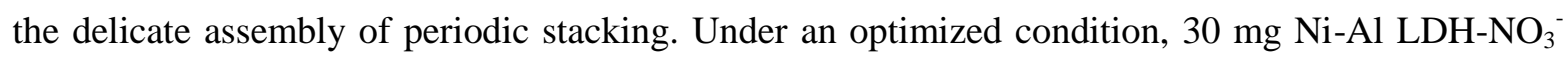
was exfoliated in $100 \mathrm{~mL}$ formamide as the $\mathrm{LDH}$ stock solution. $15 \mathrm{mg} \mathrm{GO}$ was exfoliated in $50 \mathrm{~mL}$ DMF and then diluted to $100 \mathrm{~mL}$ formamide/DMF (1:1) solution as the GO stock solution. $200 \mathrm{~mL}$ formamide was stirred in a beaker. The two stock solutions were simultaneously fed into the $200 \mathrm{~mL}$ formamide solution at the same speed of $1.5 \mathrm{~mL} / \mathrm{min}$. After the process, the flocculated product could be centrifuged, washed and freeze-dried. The as-prepared Ni-Al LDH/GO superlattice material can be reduced with hydrazine vapor. The XRD patterns (Fig. 3a) show both Ni-Al LDH/GO and $\mathrm{Ni}-\mathrm{Al} \mathrm{LDH} / \mathrm{rGO}$ have a vertical superlattice period of about $1.1 \mathrm{~nm}$, which is somewhat smaller than the sum of the AFM thickness of LDH $(\sim 0.8 \mathrm{~nm})$ and GO $(\sim 0.7 \mathrm{~nm})$ due to the stress from electrostatic attraction and the fact that AFM thickness contains the adsorption layer. ${ }^{3,6}$ The clear diffraction peaks of $(002)$ at about $16.2^{\circ}(0.54 \mathrm{~nm})$ provides unambiguous evidence for the formation of alternately stacked superlattice structure. This result demonstrates our protocol is effective considering that this (002) peak was not observed in those products when exfoliated LDH and GO were mixed without suitable control. ${ }^{17}$ Meanwhile, the high-resolution TEM (HRTEM) image (Fig. 3b) of Ni-Al LDH/GO confirms that each period with two alternating layers contributes to the thickness of $1.1 \mathrm{~nm}$, which rules out the possibility of anions/solvent insertion. The diffraction rings of both LDH and GO are presented in the SAED pattern of Ni-Al LDH/GO (Fig. 3c). HADDF-mapping images of Ni-Al LDH/GO superlattice (Fig. S2) show the existence of Ni, Al, C and $\mathrm{O}$. The phase and morphology of the superlattice materials before and after hydrazine vapor treatment is similar (XRD in Fig. 3a and TEM images in Fig. S3a). As expected, the color of Ni-Al LDH/GO changes from brown to dark after the hydrazine reduction (Fig. S3a), which means improved electrical conduction. Using Gaussian fitting for the Raman spectra, the ratio of D peak and $\mathrm{G}$ peak $\left(\mathrm{S}_{\mathrm{D}} / \mathrm{S}_{\mathrm{G}}\right)$ also decreased from 1.57 for Ni-Al LDH/GO to 1.13 for Ni-Al LDH/rGO (Fig. $\mathrm{S} 3 \mathrm{~b}$ and Table $\mathrm{S} 1$ ), indicating that the reduced material has more in-plane $\mathrm{sp}^{2}$ bonding, i.e. higher degree of graphitization and better electronic conduction. These results indicate that the as-prepared $\mathrm{Ni}-\mathrm{Al} \mathrm{LDH} / \mathrm{GO}$ can be reduced to $\mathrm{Ni}-\mathrm{Al} \mathrm{LDH} / \mathrm{rGO}$ while maintaining its superlattice structure.

For the two kinds of exfoliated sheets with opposite charges, the face-to-face electrostatic attraction needs to be dominant in order to form periodically stacked superlattice instead of randomly mixed composites. As we have mentioned, it should be more difficult for Ni-Al LDH to form an ideal anisotropic 2D superlattice structure compared to most other LDHs like Co-Al LDH because of the smaller lateral size of exfoliated Ni-Al LDH sheets. ${ }^{19,22,23}$ Achieving controllability of the 
assembly process of $\mathrm{Ni}-\mathrm{Al} \mathrm{LDH} / \mathrm{GO}$ superlattice is significant in that it provides general guideline on the assembly of other $2 \mathrm{D}$ superlattice materials. The influence of various experimental parameters in the protocol was investigated to better understand our synthetic strategy. First, the solvent needs optimizing. For common LDHs intercalated with $\mathrm{NO}_{3}{ }^{-}$with no special modification, only formamide can exfoliate LDHs effectively as far as we know. ${ }^{4,}{ }^{14}$ However, there are more candidates for exfoliating GO. Unlike the assembly of other $\mathrm{LDH} / \mathrm{GO}$ or $\mathrm{LDH} /$ polymer superlattice, we find that using water to disperse $\mathrm{GO}$ will result in the failure of $\mathrm{Ni}-\mathrm{Al} \mathrm{LDH} / \mathrm{GO}$ superlattice formation during the co-feeding process (Fig. S4a). Water is known to restore the interlayer hydrogen bonding network of LDH and would result in restacking of LDH directly. ${ }^{14}$ We choose DMF to exfoliate GO and then dilute it with formamide as the GO stock solution. DMF has a similar chemical structure as formamide and it can exfoliate GO. ${ }^{25}$ When a small amount of water is introduced into the GO stock solution $\left(\mathrm{V}_{\mathrm{H} 2 \mathrm{O}}: \mathrm{V}_{\mathrm{DMF}}: \mathrm{V}_{\text {formamide }}=2: 48: 50\right)$, the XRD pattern of obtained superlattice material shows poorer crystallization (Fig.4a). When more water is introduced $\left(\mathrm{V}_{\mathrm{H} 2 \mathrm{O}}: \mathrm{V}_{\mathrm{DMF}}: \mathrm{V}_{\text {formamide }}=10: 40: 50\right)$, the basal spacing of obtained product is $0.85 \mathrm{~nm}$ and no (002) diffraction of superlattice can be observed (Fig. 4a). This result indicates the inclusion of water would result in the formation of more random $\mathrm{LDH}+\mathrm{GO}$ nanocomposite instead of periodic $(\mathrm{LDH} / \mathrm{GO})_{n}$ superlattice. Noteworthy, once the superlattice materials are successfully assembled, its structure could endure subsequent water washing. Therefore, dried powder can be recovered without aggregation by freeze-drying.

Second, the key to the success of our co-feeding protocol lies in the mixing process, i.e. the two stock solutions are slowly pumped into another $200 \mathrm{~mL}$ formamide solvent so that the reaction proceeds under a gentle (diluted) condition. When the Ni-Al LDH is pumped directly into GO solution, the diffraction peak of obtained product shows most Ni-Al LDH re-stacks with itself and only small amount of alternately stacked superlattice exists (Fig. S4b). When the Ni-Al LDH solution is quickly poured into the GO solution, no superlattice material can be observed (Fig. S4b). This indicates that the co-feeding protocol is a very powerful method to control the nucleation and growth of $(\mathrm{LDH} / \mathrm{GO})_{n}$ in self-assembly.

Third, the influence of relative pumping rate of the two stock solution is investigated. When the feeding rate of the two solution is balanced or when the Ni-Al LDH solution is pumped at a slightly faster rate $(1.8 \mathrm{~mL} / \mathrm{min} \mathrm{Ni}-\mathrm{Al} \mathrm{LDH}$ vs. $1.5 \mathrm{~mL} / \mathrm{min} \mathrm{GO}), \mathrm{Ni}-\mathrm{Al} \mathrm{LDH} / \mathrm{GO}$ superlattice with good quality can be obtained (Fig. S4c). But when the GO solution is pumped faster $(1.5 \mathrm{~mL} / \mathrm{min} \mathrm{Ni}-\mathrm{Al}$ $\mathrm{LDH}$ vs. $1.8 \mathrm{~mL} / \mathrm{min} \mathrm{GO}$ ), the $\mathrm{Ni}-\mathrm{Al} \mathrm{LDH} / \mathrm{GO}$ becomes poorly crystallized although it still possesses the alternately stacked structure (Fig. S4c). This indicates it would be better to make the $\mathrm{LDH}$ phase in excess since exact ratio control of the two materials is challenging. 
Fourth, the ratio of both materials is also important. $30 \mathrm{mg} \mathrm{Ni-Al} \mathrm{LDH} \mathrm{vs.} 15 \mathrm{mg}$ GO is an optimized ratio. When either of $\mathrm{Ni}-\mathrm{Al} \mathrm{LDH}$ or GO is deficient, Ni-Al LDH phase with basal spacing of 0.78 0.92 nm would form (Fig. S4d and Fig. S4e). It is noteworthy that when GO is extremely excessive (30 mg Ni-Al LDH vs. $30 \mathrm{mg} \mathrm{GO}$ ), the assembled product shows a broad diffraction peak at about $0.92 \mathrm{~nm}$ and no (002) peak can be observed (Fig. 4f). If treated with the same hydrazine vapor process, this phase would convert into a regular LDH/GO composite phase showing diffraction feature of hydrotalcite. This shows that well-defined (001) and (002) diffraction peaks are necessary to differentiate $(\mathrm{LDH} / \mathrm{GO})_{n}$ superlattice from $\mathrm{LDH}+\mathrm{GO}$ nanocomposite (which would also show larger basal spacing due to ion/solvent insertion). ${ }^{17}$ In brief, the following play critical roles in the assembly process: solvent system should be optimized to exclude water, a co-feeding method in dilute solution can be used to provide a mild reaction condition, a balanced pumping speed is favored, and the mass ratio $\mathrm{m}_{\mathrm{Ni}-\mathrm{Al} \mathrm{LDH}}: \mathrm{m}_{\mathrm{GO}}$ should be about 2:1. The theoretical mass ratio can be derived based on an area-matching model (Table $\mathrm{S} 2$ ). We estimate the $\mathrm{m}_{\mathrm{Ni}-\mathrm{Al} \mathrm{LDH}}: \mathrm{m}_{\mathrm{GO}}$ is favored to be within $1.8 \sim 2.4$. This value is smaller than previously calculated $3 \sim 3.3$ for Co-Al (or Co-Ni) LDH/GO and about 5.1 for $\mathrm{Ni}-\mathrm{Fe} / \mathrm{GO},{ }^{3,6}$ because the mass we used is based on the dried GO while those literature values were calculated based on the graphite used to synthesize GO. Meanwhile, using DMF to exfoliate GO is not as efficient as using water, which would result in some mass loss of GO (Table S2).

Our synthetic strategy is expected to be versatile. We have chosen a more complex ternary $\mathrm{Ni}-\mathrm{Co}-\mathrm{Al} \mathrm{LDH}$ system to test the transferability. Under similar experimental condition but using $\mathrm{Ni}-\mathrm{Co}-\mathrm{Al} \mathrm{LDH}$ as precursor, the assembled Ni-Co-Al/GO also has the alternately stacked superlattice structure (See XRD in Fig. S5a and HAADF-mapping image in Figure S5b).

\subsection{Hybrid supercapacitor demonstration}

To increase the energy density of supercapacitor without sacrificing power density, a promising way is to increase the cell voltages by developing asymmetric supercapacitors, which possess a battery-type Faradaic electrode coupled with an electric double-layer (EDL) capacitive electrode in an aqueous electrolyte that has high ionic conductivities. ${ }^{26-29}$ In the search of new electrode materials, two-dimensional solids are particularly interesting due to their large electrochemically active surfaces. ${ }^{30,31} \mathrm{Ni}-\mathrm{Al} \mathrm{LDH}$ is believed to store electrochemical energy based on the oxidation and reduction of $\mathrm{Ni}$ (the redox of the brucite layers based on $\alpha-\mathrm{Ni}(\mathrm{OH})_{2} / \gamma-\mathrm{NiOOH}$ conversion). ${ }^{18}$ As we mentioned above, the structure of $\mathrm{Ni}-\mathrm{Al} \mathrm{LDH} / \mathrm{GO}$ superlattice can endure water washing, freeze-drying and hydrazine vapor reduction. Our material in powder form is more compatible with existing industrial plant processes to fabricate energy storage devices than previously reported 
$\mathrm{LDH} /$ graphene or $\mathrm{LDH} /$ polymer superlattices which were only used in the wet state. ${ }^{3,6,15}$

The electrochemical performance is first evaluated in a standard three-electrode system. Compared with the original $\mathrm{Ni}-\mathrm{Al} \mathrm{LDH}$, the cyclic voltammetry (CV) curves of $\mathrm{Ni}-\mathrm{Al} \mathrm{LDH} / \mathrm{rGO}$ have larger areas in the redox peaks at various scan rates, indicating an improved electrochemical activity (Fig. S6a and S6b). The Ni-Al/rGO superlattice shows a longer discharge curve than that of bare Ni-Al LDH (Figure S6c and S6d). At 1 A/g, the specific capacity of the Ni-Al/rGO superlattice is about 2 times that of $\mathrm{Ni}-\mathrm{Al} \mathrm{LDH}(129 \mathrm{Ah} / \mathrm{kg}$ vs. $60 \mathrm{Ah} / \mathrm{kg})$. The constant charge/discharge cycling test $(5 \mathrm{~A} / \mathrm{g}$, or $1.5 \mathrm{~min}$ full discharge) show that the specific capacity of $\mathrm{Ni}-\mathrm{Al} \mathrm{LDH} / \mathrm{rGO}$ shows a little increase in the first 0 500 cycles, decreased rapidly in about 1000 5000 cycle, and finally showed little decrease in 5000 10000 cycles (Fig. S6f). This "activation-rapid decay-stabilization" cycling behavior is also observed for other redoxable single layered hydroxides like $\alpha-\mathrm{Ni}(\mathrm{OH})_{2}$ and $\alpha-\mathrm{Co}(\mathrm{OH})_{2}{ }^{23}$ After 10000 cycles, the Ni-Al/rGO superlattice can still maintain about $72.7 \%$ of its initial capacity (Fig. S6f).

To demonstrate the feasibility of $\mathrm{Ni}-\mathrm{Al} \mathrm{LDH} / \mathrm{rGO}$ superlattice materials for practical applications, we have fabricated an asymmetric supercapacitor with high mass loading using activated carbon (AC) as the negative electrode ( $5 \mathrm{mg} \mathrm{Ni-Al/rGO}$ vs. $\sim 15 \mathrm{mg} \mathrm{AC}$ ). The $\mathrm{CV}$ and chronopotentiometry curves of AC and the full supercapacitor are given in Fig. 4a and 4b. For a device loaded with 4.79 $\mathrm{mg} \mathrm{Ni}-\mathrm{Al} / \mathrm{rGO}$ and $15.48 \mathrm{mg} \mathrm{AC}$, the specific capacitances (Fig. S7c) are calculated to be 39.55 to $19.38 \mathrm{~F} / \mathrm{g}$ (based on $m_{\mathrm{Ni}-\mathrm{Al} / \mathrm{rGO}}+m_{\mathrm{AC}}$ ) when the discharge current increases from 5 to $50 \mathrm{~mA}$ (current density of 1.04 to $10.4 \mathrm{~A} / \mathrm{g}$ based on $m_{\mathrm{Ni}-\mathrm{Al} / \mathrm{rGO}}$ ). The Ragone plots (Fig. S7d) are calculated by integrating the voltage-discharge time profile (Fig. 4b). The energy density ranges from 8.07 to 15.42 $\mathrm{Wh} / \mathrm{kg}$ when the powder density is set from 3.42 to $0.23 \mathrm{~kW} / \mathrm{kg}$. The cycling behavior of the full device also experiences "activation-rapid decay-stabilization" stages (Fig. 4c). After cycling at 10 $\mathrm{mA}\left(2.09 \mathrm{~A} / \mathrm{g}\right.$ based on $\left.m_{\mathrm{Ni}-\mathrm{A} l / \mathrm{rGO}}\right)$, the capacity retention is $67.4 \%$, slightly lower than that of Ni-Al $\mathrm{LDH} / \mathrm{rGO}$ tested in three-electrode system. The specific capacitance and energy density of the asymmetric supercapacitor fall into the range of a typical traditional supercapacitor. The contribution of the improved positive electrode is limited because the main contribution of mass comes from the AC in the negative electrode. A higher performance can be expected if a better negative electrode is developed.

The (Ni-Al LDH/rGO) $)_{n}$ superlattice shows obvious improvement compared with the pristine $\mathrm{Ni}-\mathrm{Al} \mathrm{LDH}$. While the absolute performance of this material in alkaline hybrid supercapacitor is not stellar, in comparing with other materials one should be aware of the following pitfalls. First, the performance of battery-type LDHs (based on specific capacity, $\mathrm{Ah} / \mathrm{kg}$ ) are often mistakenly 
evaluated as pseudocapacitive materials (based on specific capacitance, F/g). The reported "specific capacitance" could range from 400 to $3500 \mathrm{~F} / \mathrm{g}$ based on different calculation methods, that are highly sensitive to the potential range chosen. ${ }^{32-36}$ Second, the deviation in specific capacity can be up to $20 \times$ because of different mass loading. ${ }^{32}$ Third, some ultra-high specific capacity measured in nanostructured materials may be a result of ultra-low tap density of the powders. ${ }^{37}$ In industrial settings, the areal loading $\left(\sim 5 \mathrm{mg} / \mathrm{cm}^{2}\right.$ here) as well as the packing density (related to the thickness of the slurry) are critical for cell-pack level cost/performance. To show that our full-cell device works well, we use it to drive an LED board. As a proof of concept, we assembled four CR2025 coin cells in series to drive a circuit board with 22 parallel LEDs (12 red and 10 yellow) in series, with an additional $32 \Omega$ electrical resistor for limiting the current (Fig.4d). Theoretically, two or three cells should be enough to provide sufficient voltage if we use a smaller circuit-protecting resistor.

\section{Conclusion}

We have developed a general and novel synthesis strategy to fabricate true $(\mathrm{Ni}-\mathrm{Al} \mathrm{LDH} / \mathrm{rGO})_{n}$ and (Ni-Co-Al LDH/rGO) $)_{n}$ superlattice materials by optimizing the solvent and dispersion, pumping speed of stock solutions and mass ratio of two layered materials in the co-feeding mode. Such well-controlled, low-cost, high-yield process for self-assembling periodic stacks of 2D charged sheets opens new avenues to investigate vertically stacked artificial 2D materials and their applications in electrochemical energy storage and electrocatalysis.

\section{ASSOCIATED CONTENT}

\section{Supporting Information:}

This information is available free of charge via the Internet.

\section{- ACKNOWLEDGEMENTS}

This work was supported by the National Natural Science Foundation of China (51271169), the Key Science and Technology Innovation Team of Zhejiang Province under grant number 2010R50013, the Program for Innovative Research Team in University of Ministry of Education of China (IRT13037), and the Zhejiang Provincial Natural Science Foundation of China (LY16B030005). Xiang Ge acknowledges the support from China Scholarship Council (CSC). ZYY and JL acknowledge support by NSF DMR-1410636 and the Center for Excitonics, an Energy Frontier Research Center funded by the U.S. Department of Energy, Office of Science, Basic Energy Sciences under Award No. DE-SC0001088.

\section{- AUTHOR INFORMATION}

\section{Corresponding Authors}


*(C.D. Gu) E-mail: cdgu@zju.edu.cn

*(J. Li) E-mail: 1iju@mit.edu

Note

The authors declare no competing financial interest. 


\section{- REFERENCES}

1. Wang, Q.; O'Hare, D. Chem. Rev. 2012, 112, (7), 4124-4155.

2. Forticaux, A.; Dang, L.; Liang, H.; Jin, S. Nano Lett. 2015, 15, (5), 3403-3409.

3. Ma, R.; Liu, X.; Liang, J.; Bando, Y.; Sasaki, T. Advanced Materials 2014, 26, (24), 4173-4178.

4. Ma, R.; Sasaki, T. Adv. Mater. 2010, 22, (45), 5082-5104.

5. Ma, R.; Sasaki, T. Acc. Chem. Res. 2014, 48, (1), 136-143.

6. Ma, W.; Ma, R.; Wang, C.; Liang, J.; Liu, X.; Zhou, K.; Sasaki, T. ACS Nano 2015, 9, (2), 1977-1984.

7. Vialat, P.; Mousty, C.; Taviot-Gueho, C.; Renaudin, G.; Martinez, H.; Dupin, J.-C.; Elkaim, E.; Leroux, F. Adv. Funct. Mater. 2014, 24, (30), 4831-4842.

8. Cheng, J.; Zhang, J.; Liu, F. RSC Adv. 2014, 4, (73), 38893-38917.

9. Cheng, J. P.; Liu, L.; Zhang, J.; Liu, F.; Zhang, X. B. J. Electroanal. Chem. 2014, 722-723, (0), 23-31.

10. Ge, X.; Gu, C. D.; Wang, X. L.; Tu, J. P. J. Mater. Chem. A 2014, 2, (40), 17066-17076.

11. Hu, Z.-A.; Xie, Y.-L.; Wang, Y.-X.; Xie, L.-J.; Fu, G.-R.; Jin, X.-Q.; Zhang, Z.-Y.; Yang, Y.-Y.; Wu, H.-Y. J. Phys. Chem. C 2009, 113, (28), 12502-12508.

12. Ge, X.; Gu, C. D.; Wang, X. L.; Tu, J. P. J. Phys. Chem. C 2014, 118, (2), 911-923.

13. Nicolosi, V.; Chhowalla, M.; Kanatzidis, M. G.; Strano, M. S.; Coleman, J. N. Science 2013, 340, (6139).

14. Ma, R.; Liu, Z.; Li, L.; Iyi, N.; Sasaki, T. J. Mater. Chem. 2006, 16, (39), 3809-3813.

15. Zhao, J.; Xu, S.; Tschulik, K.; Compton, R. G.; Wei, M.; O'Hare, D.; Evans, D. G.; Duan, X. Adv. Funct. Mater. 2015, 25, (18), 2745-2753.

16. Huang, Z.; Wang, S.; Wang, J.; Yu, Y.; Wen, J.; Li, R. Electrochim. Acta 2015, 152, (0), 117-125.

17. Hu, J.; Lei, G.; Lu, Z.; Liu, K.; Sang, S.; Liu, H. Chem. Commun. 2015, 51, 9983-9986.

18. Wimalasiri, Y.; Fan, R.; Zhao, X. S.; Zou, L. Electrochim. Acta 2014, 134, 127-135.

19. Liu, Z.; Ma, R.; Ebina, Y.; Iyi, N.; Takada, K.; Sasaki, T. Langmuir 2007, 23, (2), 861-867.

20. Osada, M.; Sasaki, T. Polym J 2014, 47, (2), 89-98.

21. Marcano, D. C.; Kosynkin, D. V.; Berlin, J. M.; Sinitskii, A.; Sun, Z.; Slesarev, A.; Alemany, L. B.; Lu, W.; Tour, J. M. ACS Nano 2010, 4, (8), 4806-4814.

22. Liu, Z.; Ma, R.; Osada, M.; Iyi, N.; Ebina, Y.; Takada, K.; Sasaki, T. J. Am. Chem. Soc. 2006, 128, (14), 4872-4880.

23. Gu, C. D.; Ge, X.; Wang, X. L.; Tu, J. P. J. Mater. Chem. A 2015, 3, (27), 14228-14238.

24. Stankovich, S.; Dikin, D. A.; Piner, R. D.; Kohlhaas, K. A.; Kleinhammes, A.; Jia, Y.; Wu, Y.; Nguyen, S. T.; Ruoff, R. S. Carbon 2007, 45, (7), 1558-1565.

25. Paredes, J.; Villar-Rodil, S.; Martinez-Alonso, A.; Tascon, J. Langmuir 2008, 24, (19), 10560-10564.

26. Lu, X.; Yu, M.; Wang, G.; Zhai, T.; Xie, S.; Ling, Y.; Tong, Y.; Li, Y. Adv. Mater. 2013, 25, (2), 267-272.

27. Xia, X.; Chao, D.; Fan, Z.; Guan, C.; Cao, X.; Zhang, H.; Fan, H. J. Nano Lett. 2014, 14, (3), 1651-1658.

28. Peng, S.; Li, L.; Wu, H. B.; Madhavi, S.; Lou, X. W. D. Advanced Energy Materials 2015, 5, (2). 1401172

29. Liu, J.; Zhang, L.; Wu, H. B.; Lin, J.; Shen, Z.; Lou, X. W. D. Energy \& Environmental Science 2014, 7, (11), 3709-3719.

30. Chhowalla, M.; Shin, H. S.; Eda, G.; Li, L.-J.; Loh, K. P.; Zhang, H. Nature chemistry 2013, 5, (4), 263-275.

31. Ghidiu, M.; Lukatskaya, M. R.; Zhao, M.-Q.; Gogotsi, Y.; Barsoum, M. W. Nature 2014, DOI:10.1038/nature13970.

32. Li, H. B.; Yu, M. H.; Wang, F. X.; Liu, P.; Liang, Y.; Xiao, J.; Wang, C. X.; Tong, Y. X.; Yang, G. W. Nat Commun 2013, 4, 1894. 
33. Jiang, W.; Yu, D.; Zhang, Q.; Goh, K.; Wei, L.; Yong, Y.; Jiang, R.; Wei, J.; Chen, Y. Adv. Funct. Mater. 2015, 25, (7), 1063-1073.

34. Bastakoti, B. P.; Huang, H.-S.; Chen, L.-C.; Wu, K. C.-W.; Yamauchi, Y. Chem. Commun. 2012, 48, (73), 9150-9152.

35. Du, H.; Jiao, L.; Cao, K.; Wang, Y.; Yuan, H. ACS Appl. Mater. Interfaces 2013, 5, (14), 6643-6648.

36. Jiang, H.; Zhao, T.; Li, C.; Ma, J. J. Mater. Chem. 2011, 21, (11), 3818-3823.

37. Gogotsi, Y.; Simon, P. Science Magazine 2011, 334, 917-918. 


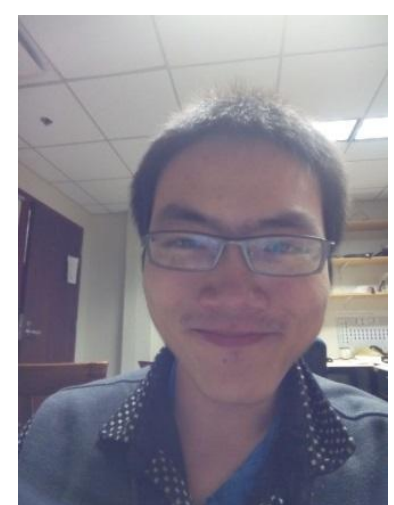

Xiang Ge (Research ID: N-6879-2015) is persuing his Ph. D. under the supervision of Dr. Changdong Gu since 2012 in the School of Materials Science \& Engineering in Zhejiang University (ZJU), China. In 2015, financed with the scholarship from China Scholarship Council (CSC), he went to Massachusetts Institute of Technology (MIT) as a visiting student supervised by Dr. Ju Li. His research interest includes the synthesis of redoxable layered materials and their derivatives used in energy and environment related applications including battery, supercapacitor, electro-catalysis and adsorption.

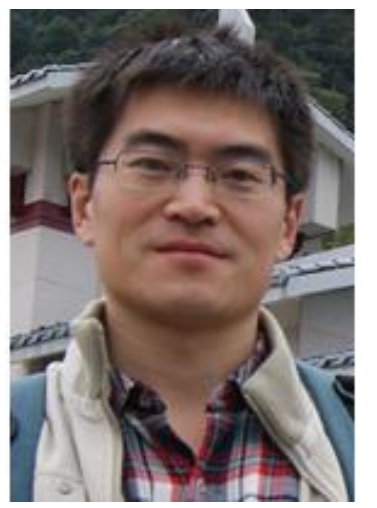

Dr. Changdong Gu (ResearcherID: B-3299-2008) received his Ph.D degree in material science from Jilin University in 2007 supervised by Prof. J. S. Lian. He worked at the Department of Mechanical Engineering, HKUST from 2007 to 2009 as a Research Associate in Prof. T.-Y. Zhang's group. Then he joined School of Materials Science and Engineering, Zhejiang University as an Associate Professor. His research work focuses on energy storage materials, surface and coatings technoloy, and novel application of deep eutectic solvents in electrochemistry. [Homepage: http://mypage.zju.edu.cn/gcd].

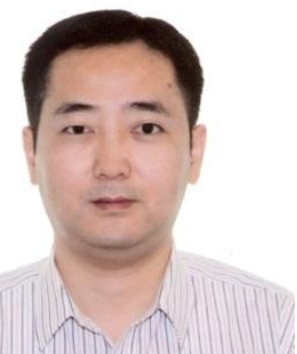

Zongyou Yin studied at Jilin University in China for his B.E. and M.S., and completed his Ph.D. followed by a research fellow career at Nanyang Technological University (NTU) in Singapore. At the beginning of year 2014, he moved to Massachusetts Institute of Technology (MIT) as a postdoctoral associate. His research interests include the low dimensional nanomaterials and their applications for clean energy, environment and optoelectronics. 


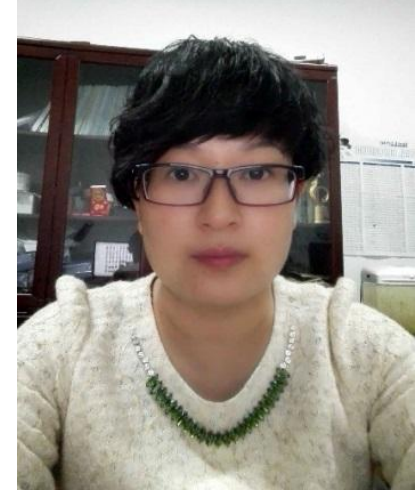

Dr. Xiuli Wang is an associate professor at School of materials Science and Engineering, Zhejiang University. She received Ph.D degree supervised by Prof. Jiangping Tu in 2006 from Zhejiang University. Then she joined Prof. Jiangping Tu's group as a faculty of Zhejiang University. She joined Associate Prof.Ting Yu's group as a post-doctor at Nanyang Technological University of Singapore. Her research work focus on the electrode materials for lithium ion batteries and battery technology, materials surface modification technology. Since 2006, Dr.Wang has published over 170 SCI articles (h-index:39).

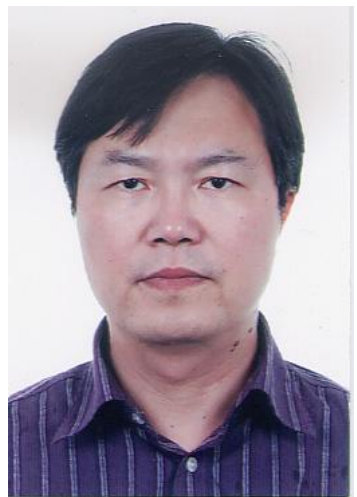

Jiang-ping Tu received his BS degree in Shanghai Jiaotong University in 1985. He obtained his MS in 1991 and Ph. D in 1994 at Zhejiang University, China. From 1998 to 2000, he worked as a Japan Society for the Promotion of Science (JSPS) research fellow. Since 2000 he is a full professor at Zhejiang University. His main research interests are materials for Li-ion and other rechargeable batteries, nancomposite films, coatings and physical metallurgy. He has 410 publications cited by SCI (H-Index =57) and 89 patents, and gains four prizes of science and technology of government. He is a 2015 Thomson Reuters Highly Cited Researcher.

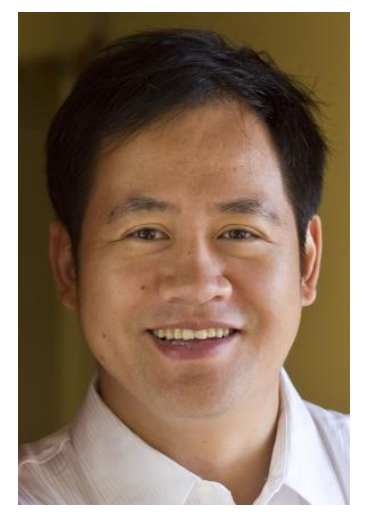

$\mathrm{Ju} \mathrm{Li}$ is BEA Professor of Nuclear Science and Engineering and Professor of Materials Science and Engineering at MIT. His group (http://Li.mit.edu) performs computational and experimental research on mechanical properties of materials, and energy storage and conversion. $\mathrm{Ju}$ is a recipient of the 2005 Presidential Early Career Award for Scientists and Engineers, 2006 MRS Outstanding Young Investigator Award, and 2007 TR35 award from Technology Review magazine. Thomson Reuters included $\mathrm{Ju}$ in its Highly Cited Researchers list in 2014, among 147 global scientists in the Materials Science category. Ju was elected Fellow of the American Physical Society in 2014. 


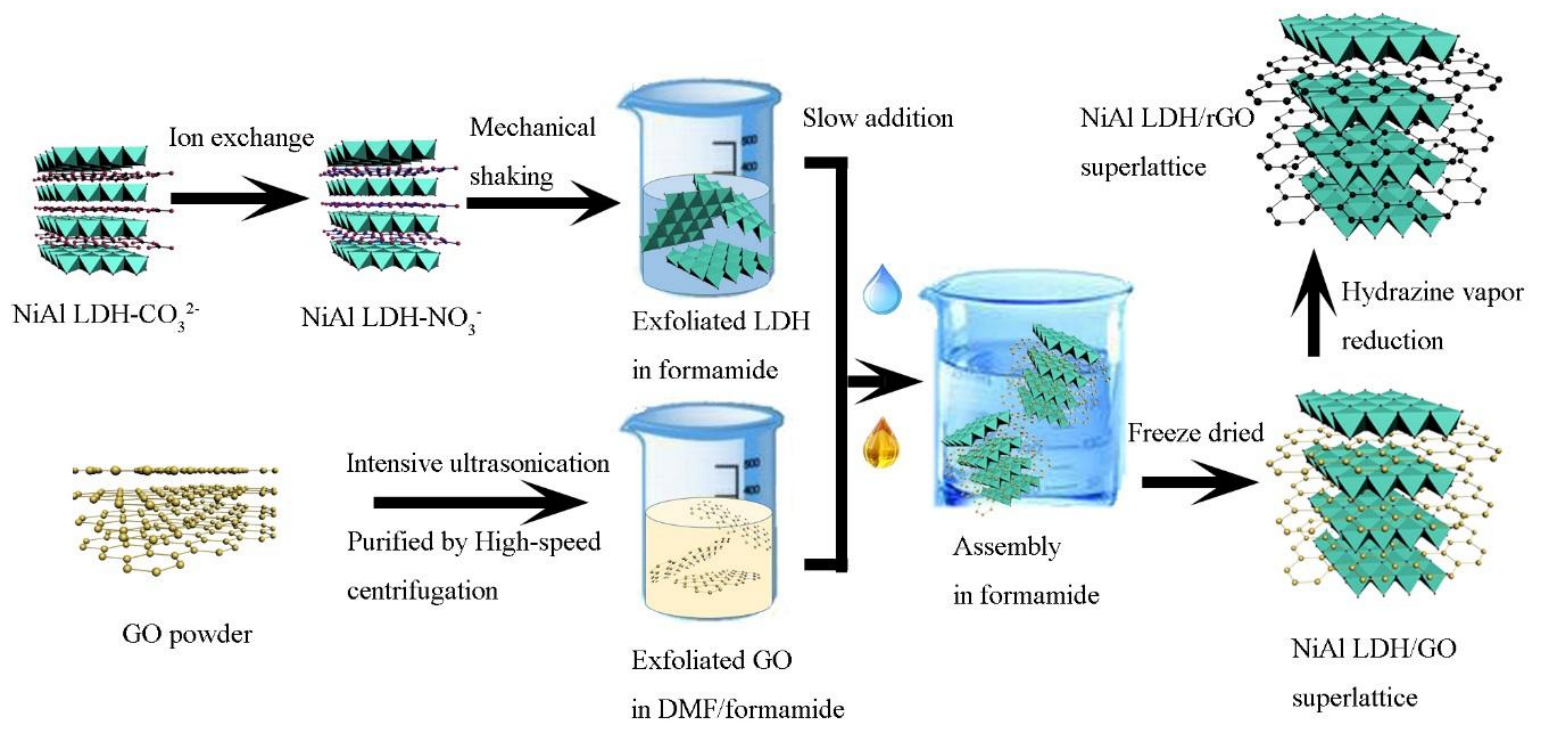

Figure 1 Schematic illustration of the preparation process of $\mathrm{Ni}-\mathrm{Al} \mathrm{LDH} / \mathrm{rGO}$ superlattice materials. 

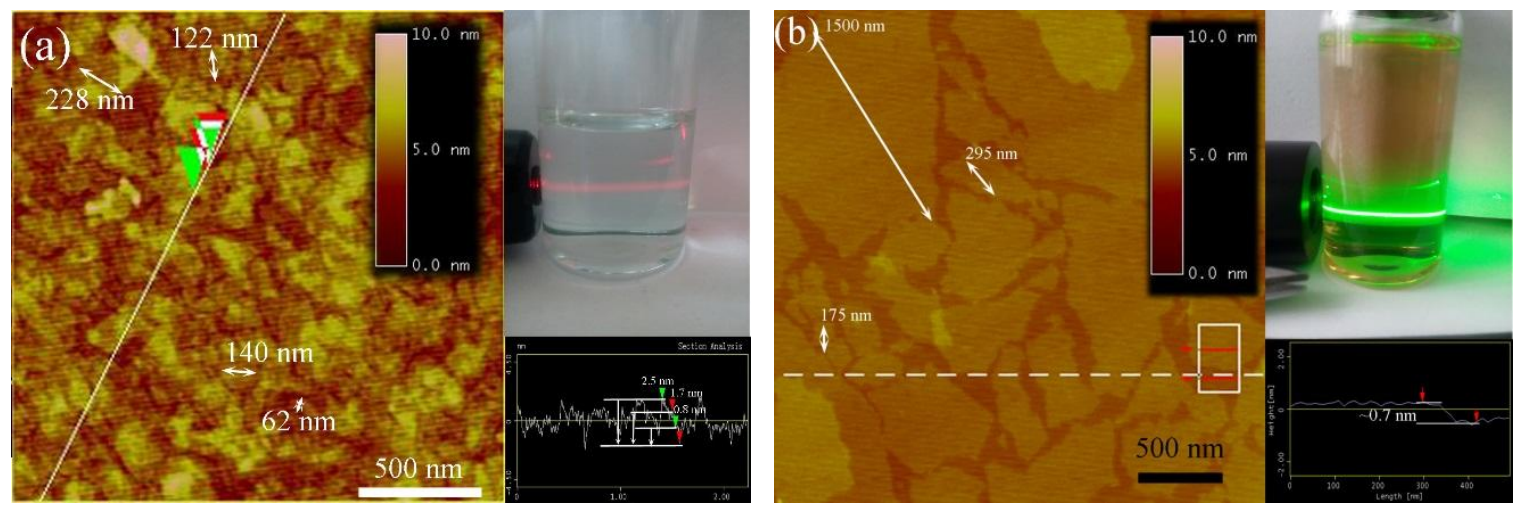

Figure 2 AFM characterization of exfoliated Ni-Al LDH (a) and exfoliated GO (b) sheets. 

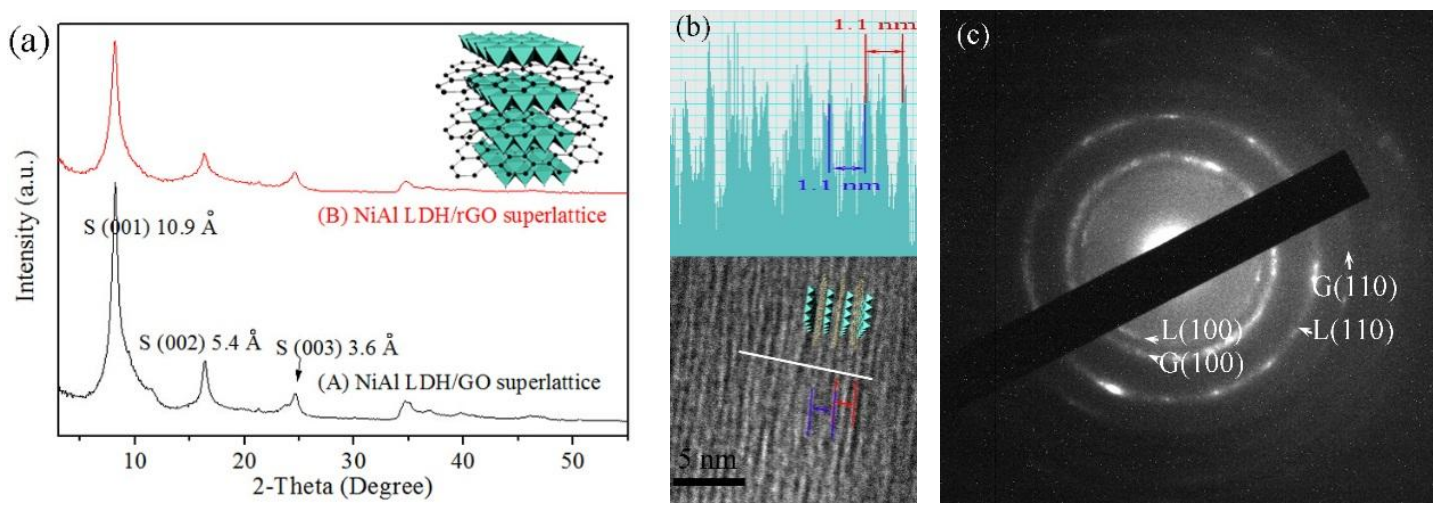

Figure 3 (a) XRD patterns of Ni-Al LDH/GO and Ni-Al LDH/rGO superlattice structures. (b) HRTEM and (c) SAED images of Ni-Al LDH/GO supperlattice structures 

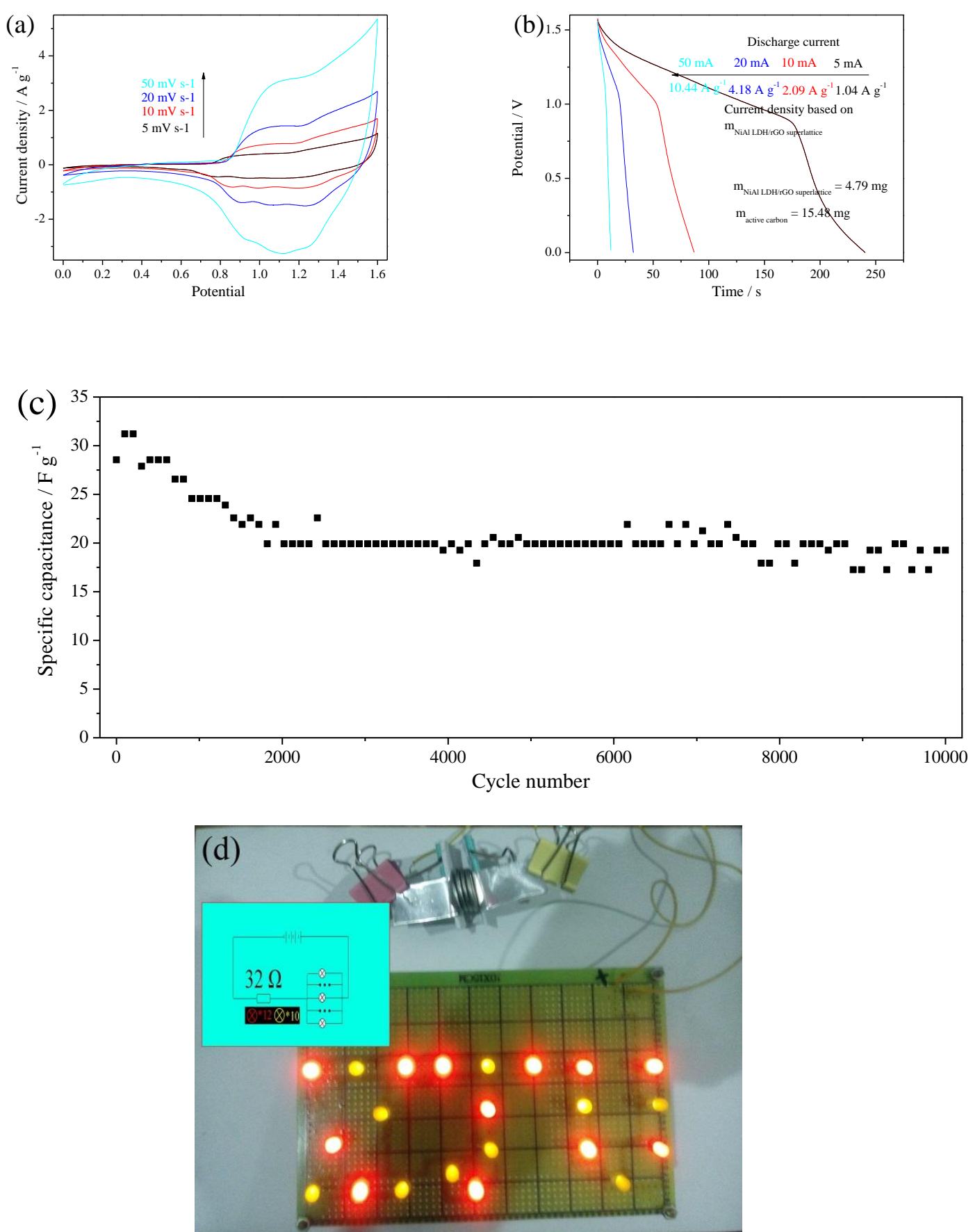

Figure 4 Demonstration of a high-loading (4.79 mg Ni-Al LDH/rGO superlattice vs. 15.48 mg AC) asymmetric supercapacitor. (a) shows the CV curves. (b) gives the chronopotentiometry curves. (c) gives the cycling performance at $10 \mathrm{~mA}\left(2.09 \mathrm{~A} \mathrm{~g}^{-1}\right.$ based on $\left.\mathrm{m}_{\mathrm{Ni}-\mathrm{Al} / \mathrm{rGO}}\right)$. The specific capacitance is calculated based on the total mass of active materials on both electrodes. (d) shows the digital picture of a circuit board with 22 parallel LEDs (12 red and 10 yellow) in series with a $32 \Omega$ current limiting resistance driven by four asymmetric device in serieFig.s. 
TABLE OF CONTENTS (TOC) IMAGE
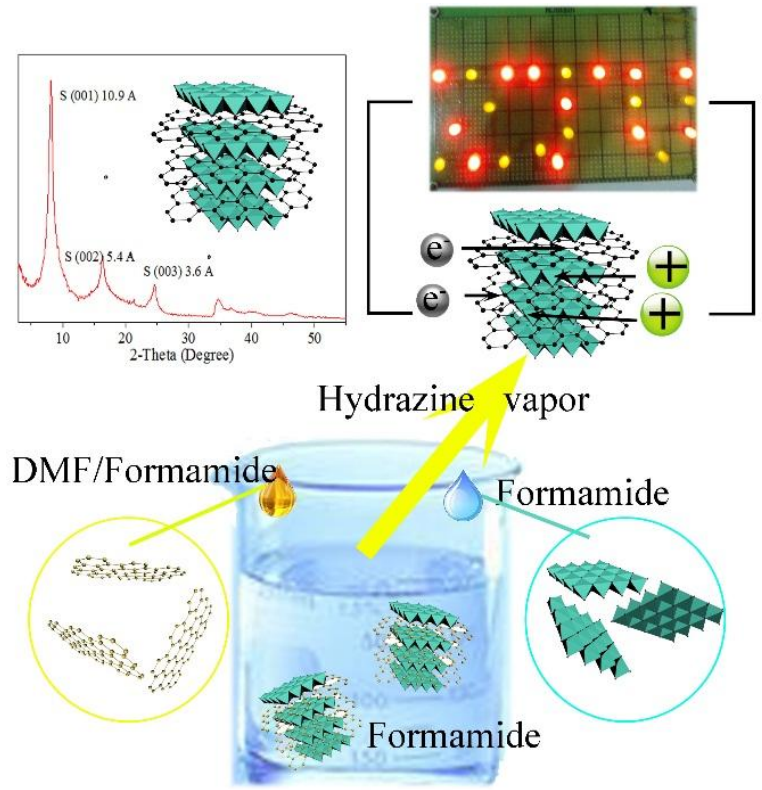